\title{
26
}

\section{The Use of Information Technology in Educational Counselling: Applications for High School Counsellors}

\author{
Y. J. Katz and B. Offir \\ School of Education, Bar-Ilan University, Ramat-Gan 52900, Israel
}

\begin{abstract}
Information Technology (IT) is a universally accepted educational instrument designed to increase the effectiveness and efficiency of the educational system. Computers are used for instruction as well as learning in the school system. An increasing variety of courseware, both "traditional" and "open-ended", is available for teachers and pupils teaching and studying different subjects offered by schools and empirical evidence indicates that well-planned and suitable courseware contributes positively to the cognitive and affective needs of pupils.

However, in addition to the utilisation of IT for teaching and learning, computers can be effectively used for educational counselling. Data storage and retrieval that provide counsellors with up to date information on pupils in need of counselling can dramatically increase the efficiency of the counselling process. The effectiveness of Student Selection and Placement, Diagnostics, Reports to Parents and Teachers, School Faculty Consultation, and Statistical Data Analysis (important functions carried out by educational counsellors) can be significantly improved through the use of IT. It is suggested that educational counsellors investigate the possibility of routinely utilising the school computer in their work in order to improve counsellor effectiveness and efficiency.
\end{abstract}

Keyword codes: K.3.m1, K.3.m2, K.3.m3, K.3.m4, K.3.m5, K.3.m6, K.3.m7

Keywords: Information Technology, Educational Counselling, Student Selection and Placement, Diagnostics, Reports to Parents and Teachers, School Faculty Consultation, Statistical Data Analysis.

\section{COMPUTERS AND EDUCATION}

Robinson [1], in summing up almost twenty years of experience regarding the use of computers in the educational system, declared that it is now commonly accepted that computers should play a central role in education. Other commentators, however, recognised that the educational impact of computers within the classroom has not as yet been efficiently maximised. Moore [2] and Dunn and Ridgeway [3] confirmed that the computer has not as yet become the potent force in the educational system as expected.

Hoyle [4] predicted that the infiltration of computers into the school system may well bring about the long dreamed of educational revolution and, more specifically, the teaching and learning revolution. The computer can serve as a vehicle for improvement of students' learning [5], and for example, Hativa [6] and Mevarech [7] indicated that Israeli elementary school pupils who studied mathematics and science through the medium of computer-assisted instruction completed their study units more rapidly and accurately than their counterparts who studied the same subjects by traditional and 
older established teaching methods. However, progress has generally been limited to computer assisted instruction and learning and the most rapid developments have been made in these specific areas.

\subsection{Computers and Counselling}

Katz [8] postulated that in addition to learning and instruction there are two other major areas in education can conceivably benefit from the use of computers which may be described as the climax of technological development. These are educational administration and organisation; and auxiliary specialisations in education such as counselling. Less attention has been paid to the topic of administration and organisation or auxiliary specialisations than to learning-related topics in the literature [9]. Nevertheless, the advantages of computer utilisation in the areas of administration and counselling are evident in such modes as guidance, testing, curriculum evaluation, records and scheduling [10], as a management tool, and as a storage and retrieval device [11]. As such, the computer facilitates the work of both the school principal and the school counsellor.

Counselling, which may be described as the field in education which specializes in the concern for the individual's well-being, needs and development, is an essential facet of the total educational process. The key question that remains is whether the computer's proven contribution to learning and instruction can be successfully transferred to the field of counselling. Super [12] answered this question stating that although radical innovations such as computer assisted counselling are likely to encounter initial resistance, after familiarisation and proven effectiveness, counsellors will readily utilise computer processed information. The computer is an especially potent tool with regard to information storage and retrieval [13], counsellor accuracy and efficiency [14], improved record keeping [15], decision-making strategies [16], and counselling-related simulations [17]. Crabbs [18] added that computer assisted counselling is a cost-effective method of tabulating and manipulating data. Pogrow [19] summed up the potential of the computer for the counsellor as a delivery system designed to improve school effectiveness and efficiency by reducing personnel time that would otherwise be required for the same task.

The goal of the computerised counselling package described in this paper is to provide high school counsellors with an efficient counselling instrument. This package allows for the utilisation of all information vital to the diagnostic process and enables the counsellor to evaluate educational situations with improved accuracy. The main innovations suggested by computerised counselling are time saving, information gathering, information processing and retrieval. Information which usually takes days or even weeks to process by traditional methods is processable within minutes with the assistance of the computer. The computerised counselling package does not demand radical changes in counselling methods. The package provides the counsellor - familiar with traditional counselling procedures - with a potent and sophisticated instrument for data storage and information processing thus paving the way for improved efficiency, depth and utility in the counselling process while counselling by well-known and acceptable methods. 


\section{COMPUTER ASSISTED COUNSELLING PACKAGE}

The computerised counselling package provides the following services:

1. Student Selection and Placement

2. Diagnostics

3. Reports to Parents and Teachers

4. School Faculty Consultation

5. Statistical Data Analysis

\subsection{Student Selection and Placement}

The computerised counselling package offers the counsellor facilities for determining student selection and placement on the basis of entrance examinations, standardized achievement tests, intelligence tests, aptitude tests or other relevant measures. Students can be accepted and assigned to relevant classes on the basis of homogeneity or heterogeneity of learning levels, ethnic origin, socio-economic status or any other criteria congruent with school policy. Students can be placed in differential streams or tracks according to their ability in those subjects where streaming or tracking exists within the high school framework. This facet of computerised counselling saves much time and promotes counselling efficiency in school selection as well as class, stream or track formation.

\subsection{Diagnostics}

Diagnosis of a student will be based on the following computerised counselling information:
a. Biographical Details
b. Student Background
c. Academic Achievement
d. Standardized Testing
e. Social Standing

\subsubsection{Biographical Details}

Biographical information pertaining to all students is entered into the computer at the beginning of the school year. When a student needs counselling this information is immediately available. A biographical printout can include such data as home address, parents' educational levels, parents' occupations, number of children in family, birth order of subject and number of rooms in apartment.

\subsubsection{Student Background}

Starting from birth, all relevant developmental information is recorded on the computer. Medical, psychological and social development - based on physicians' diagnoses, intelligence, aptitude and personality tests, and teachers' as well as peers' social evaluations - is encoded and made available for retrieval should the counsellor wish to refer to the student's medical, cognitive, affective or social background for any particular reason during the course of his or her studies at school. 


\subsubsection{Academic Achievement}

All achievement-oriented information is recorded on the computer. The type of evaluation - such as class test, standardized test, homework assignment - is indicated as is the grade achieved. This information enables the counsellor to check student achievement and assess progress over a given period. In addition, the computerised counselling package allows the counsellor to compare student grades with the class or group average in any particular subject or group of subjects. The computerised package also allows for the calculation of a frequency distribution of grades, and provides a printout of students who deviate from the mean by more than one standard deviation. Students more than one standard deviation below the group mean usually have difficulty keeping pace with their peers in the academic sphere, and perhaps in other areas as well. This printout focuses attention on these low functioning students, and provides a sound basis for educational interventions.

The software package offers the option of a "Diagnosis Sheet" printout of variables considered by educational experts to be central to successful academic achievement. Key variables, such as ability, perception, thinking, learning, memory, concentration, diligence, creativity, maturity, and discipline, are readily availble from the computerised package. Teacher evaluation of these key variables can provide a clear picture of the students' problems as well as important information for the counselling process.

\subsubsection{Standardized Testing}

An important aspect of diagnostics and counseling is the assessment of student potential and achievement as compared to standardized norms. In the computerised package, the student is able to take standardized tests by computer. Immediate computerised results indicate the student's position compared to the group norm. This information allows the counsellor to suggest stream, track or class placement with a greater level of efficiency and speed. The computerised counselling package includes standardized tests such as MILTA - the Israel Group Intelligence Test [20], and the Standardized Progressive Matrices Test [21].

\subsubsection{Social Standing}

The social standing of students in their respective classes may be obtained through the medium of sociometric testing conducted with computerised counselling. The student's social status in the classroom usually adds important correlative information that can be used to verify or add to the initial diagnosis of the student's problems.

\subsection{Reports to Parents and Teachers}

Computerised counselling also allows parents to be kept informed of the students' progress and to express their opinion to an authorised faculty member. A periodical report is presented to parents and teachers on the basis of student achievement data fed into the computer over any specified period of time. When presented with a report, the parents are asked carefully to check the student's progress, to make any comments they feel appropriate and to return the signed report with comments to the class teacher.

\subsection{School Faculty Consultation}

Computerised counselling affords the counsellor the opportunity to check progress made by the teachers in their respective classes or groups. Significant differences found between parallel classes or groups for common subject matter or significant differences 
for the same classes or groups in different study topics, enable the counsellor more readily to diagnose instructional problems which typify specific teachers. Learning problems which characterise particular classes can also be diagnosed in order to assist teachers. With the computerised data the counsellor is able to advise the teachers concerned how to improve the situation in their respective classes or groups. The counsellor is also able to efficiently provide the school principal with relevant information, such as student achievement levels, inter-class differences, and instructional problems that characterise certain teachers, so that he or she may cope more readily with the implementation of school policy regarding learning and teaching effectiveness and improvement.

\subsection{Statistical Data Analysis}

Computerised counselling contains a basic statistical package including correlation, and t-test options in addition to the mean and standard deviation analyses already described. Statistical analyses of test scores at the individual and group levels enable the counsellor to make effective group and individual comparisons based on group norms. The counsellor is also able to present the principal and teachers with graphic representations of individual student and class achievements in the different subjects studied at school.

\section{CONCLUSION}

The use of the computerised counselling package at the high school level should provide the counsellor with a tool that can significantly cut down time and effort spent on data storage and retrieval as well as affording efficient information processing vital for effective counselling, consulting and coordination of student services. The counsellor could use his or her time more profitably as a result of the speedy and efficient service provided by the computerised package, without effecting any radical changes in the counselling processes traditionally used by school counsellors. Thus computerised counselling should add immeasureably to the effective contribution made by the counsellor to the students, faculty and parents. It is suggested that a comprehensive study be undertaken in order to pinpoint the advantages (and disadvantages) of computerised counselling. However, on the basis of preliminary information already available, it appears that a computerised counselling package is well worth considering as a positive technological medium that contributes to the improvement of the counselling process.

\section{REFERENCES}

1. B. Robinson, Computer assisted learning: automatons or thinking individuals, In W. Tulasiewicz and A. Adams (eds.), Teachers' expectations and teaching reality, London: Routledge, London, 1989.

2. P. Moore, Children, teachers and computers: a joint venture in learning, Journal of Curriculum Studies 19(2) (1987) 187-190.

3. S. Dunn and J. Ridgeway, Computer use during primary school teaching practice: a survey, Journal of Computer Assisted Learning 7 (1991) 7-17. 
4. E. Hoyle, Computers and education: a solution in search of a problem, In J. Megarry, D.R.F. Walker, S. Nisbet and E. Hoyle (eds.), Computers and education, Kogan Page, London, 1983.

5. S. Papert, Mindstorms: children, computers and powerful ideas, Harvester Press, Brighton, 1980.

6. N. Hativa, Computer-based drill and practice in arithmetic: widening the gap between high- and low-achieving students, American Educational Research Journal 2 (3) (1988) 366-397.

7. Z.R. Mevarech, Computer-assisted instructional methods: a factorial study within mathematics disadvantaged classrooms, Journal of Experimental Education 54 (1) (1985) 22-27.

8. Y.J. Katz, Computer assisted counselling model for the elementary school, International Journal for the Advancement of Counselling 14 (1991) 51-57.

9. A.K. Gaynor, The study of change in educational organizations, In L.L. Cunningham, W.G. Hack and R.O. Nystrand (eds.), Educational administration, McCutchan Publishing Corporation, Berkley, 1977.

10. A.R. Salisbury, Computers and education: Toward agreement on terminology, Educational Technology 11 (1971) 35-40.

11. D. Ely, Computers and the handling of information, In J. Megarry, D.R.F. Walker, S. Nisbet and E. Hoyle (eds.), Computers and education, Kogan Page, London, 1983.

12. D.E. Super, Computer assisted counseling, Teachers College Press, New York, 1970.

13. A. Das, L. Bright and M. Maby, Uses and abuses of computers in counseling, Elementary School Guidance and Counseling 18(1) (1983) 51-57.

14. J.P. Sampson, Computer-assisted testing and assessment: current status and implications for the future, Measurement and Evaluation in Guidance 15 (1983) 293-299.

15. R.C. Nelson and G.H. Krockover, Getting comfortable with computers, Elementary School Guidance and Counseling 18(1) (1983) 13-20.

16. W-C. Man and D.A. Jepsen, Effects of computer-assisted instruction in using formal decision-making strategies to choose a college major, Journal of Counseling Psychology 39(2) (1992) 185-192.

17. R.S. Sharf and M. Lucas, An assessment of a computerized simulation of counseling skills, Counselor Education and Supervision 32(4) (1993) 254-266.

18. M.A. Crabbs, Computer-assisted accountability, Elementary School Guidance and Counseling, 18(1) (1983) 41-45.

19. S. Pogrow, Education in the computer age: Issues of policy, practice and reform, Sage, Beverley Hills, 1983.

20. G. Ortar, The Israel Group Intelligence Test, Ministry of Education and Culture, Jerusalem, 1983.

21. J.C. Raven, Standard Progressive Matrices, H.K. Lewis and Company, London, 1960. 